\title{
PERSONALIDAD, TRASTORNOS DE PERSONALIDAD Y PARAMETROS
}

\author{
Vicente Pelechano ${ }^{1}$ \\ Universidad de La Laguna (Tenerife)
}

\begin{abstract}
RESUMEN
Se revisa la idea básica de personalidad y trastornos de personalidad en los dos sistemas clasificatorios predominantes dentro de la psicopatologia (CIE-10 y DSM-IV), asi como en una de sus más prestigiosas formulaciones cognitivas y se compara con la idea básica de personalidad que se encuentra recogida en un modelo estructural que parece estar creciendo (los cinco grandes), asi como en el modelo de parámetros en el que está trabajando el autor desde hace más de dos décadas. Los resultados apuntan a un desfase entre la idea de personalidad de la psicopatología y de la psicología de la personalidad $y$, por lo que se refiere a los modelos que podrían dar respuesta a las principales cuestiones planteadas, el modelo de parámetros, por su carácter estructural y de posibilidad de estudios de dinámica experimental de la personalidad y de análisis experimental poseería un mayor volumen de posibilidades de desarrollo y flexibilidad a la hora de enfrentarse a las distintas entidades psicopatológicas. El modelo es dimensional, jerárquico, flexible en cuanto a número de dimensiones y compromisos teóricos y recoge la tradición de análisis experimental extenso antes de cerrar las formulaciones teóricas, en contra de muchos de los trabajos publicados en el campo.
\end{abstract}

PALABRAS CLAVE: trastornos de personalidad, modelos estructurales de la personalidad y psicopatologia, modelo de parámetros en trastornos de personalidad.

\begin{abstract}
Central aspects of personality and personality disorders in the two main classificatory systems (ICD-10 \& DSM-IV) are revised, as well as one of the most outstanding cognitive proposals (Beck and Freeman group). These concepts are compared with the basic idea of personality of two structural models: the big-five and parameter analysis in personality psychology. The results point to the existence of a gap between the personality concepts in psychopathology, and in the psychology of personality with respect to models which could provide answers to the main questions posed. The parameter model would have greater flexibility and better prospects of development regarding different psychopathology entities due to its structural basis and enable for the study of the experimental dynamics of personality. This model is dimensional, hierarchic, flexible (with respect to number of dimensions and concrete theoretical commitments) and taking in the tradition of a broad experimental analysis before presenting a closed theory, contrary to many approaches in the domain.
\end{abstract}

KEY WORDS: personality disorders, structural models in personality and psychopathology, parameter model in personality disorders.

\footnotetext{
${ }^{1}$ Correspondencia: V. Pelechano, Dpto de Personalidad, Evaluación y Tratamientos Psicológicos. Facultad de Psicología. Campus de Guajara. La Laguna. Tenerife.
} 


\section{INTRODUCCIÓN Y PROPÓSITOS}

Los denominados "trastornos de personalidad" en la psicopatología se encuentran presentes en las dos taxonomías más divulgadas (la CIE-10 y el DSM-IV) en occidente. Desde su "presentación pública" hace poco más de una década han sido objeto de estudio y de polémica tanto acerca de su entidad como clasificación y posibilidades de intervención. Ocupa prácticamente uno de los ejes (el II, junto con el retraso mental) en el DSM-III y DSM-IV, posee una revista dedicada exclusivamente a publicar trabajos que posibiliten un mayor esclarecimiento y, a medida que se va avanzando en el número de páginas publicadas, se va complicando-oscureciendo su significación. Desde dentro mismo de la psicopatologia hay quienes lo utilizan como heurístico de la mayoria de problemas graves en psicopatologia (como hace Millon') y otros, como un elemento secundario de importancia dudosa y, por lo mismo, carente de entidad suficiente como para capitalizar un diagnóstico (con lo que ello entraña de limitaciones acerca de su explicación teórica, dedicación de investigación y posibilidades de intervención). $\mathrm{Cu}$ riosamente, desde dentro mismo de la psicología de la personalidad el tema de los trastornos (clínicos) de la personalidad no ha sido un objetivo prioritario de estudio después de su "bautismo" psicopatológico y la importancia que el pensamiento psicopatológico ha tenido en el estudio de la personalidad (por ejemplo, es vital para el modelo de H.J. Eysenck) se ha ido debilitando con el surgimiento y apli- cación del paradigma cognitivo-experimental, que ha procurado centrarse en el estudio y aportaciones provenientes de la actividad cotidiana de personas normales, a la vez que ha fomentado la confusión entre la psicología experimental con grupos criterio, la psicología diferencial y la psicopatologia experimental diferencial (Pelechano, 1993). Pensamos que este divorcio real entre psicología de la personalidad y psicopatología de la personalidad no beneficia a ninguna de las partes, aunque resulta comprensible: en la medida en que las taxonomías sean cuestión de consenso razonado, debe recogerse en ellas lo que posee ya una fuerte tradición y base empírica que se haya "implantado" o infundido y aceptado en el mundo social y profesional (ya se sabe que el consenso, como los centrismos, tiende a ser una posición conservadora en la que se huye de los modismos y las novedades que tienden a no perviviri; a la vez, la implantación-aceptación de resultados no es una resultante directa de su valor de verdad sino del paso del tiempo, poder de influencia y difusión del autor o grupo de trabajo, acceso a fuentes de poder y todo ello, con la inserción de lo nuevo dentro de un marco de progresiva sensibilización hasta pasar a ser "sentido común" del profesional) $y$, a la vez, la disciplina de psicologia de la personalidad se encuentra en uno de sus momentos dulces y bastante productivos después de la crisis en la que la intentó sepultar, por una parte el conductismo y por otra, el situacionismo cognitivo procesualista2; esta vitalidad tiende a animar a los investigadores más por

Posiblemente la figura de Millon necesite una llamada de atención puesto que no va a ser tratada en el texto y es uno de los responsables del reconocimiento de los trastornos de personalidad como entidad en los sucesivos DSM. Millon defiende una concepción de los trastornos de personalidad algo distinta al DSM-IV y CIE-10 y enraizada con el parámetro de gravedad y ha dedicado una monografia al problema (Millon, 1981), revisada poco despues. En 1994, junto a Davis (Davis y Millon, 1994), propone un esquema epistemológico interpretativo acerca de los distintos acercamientos al estudio de la personalidad que, aunque interesante como recurso heuristico, ofrece un balance no especialmente esperanzador para las teorias de la personalidad seleccionadas (predominantemente de corte norteamericano y con notorias ausencias), sirviendo esta "debilidad" para justificar el empirismo consensuado de los DSM. Mientras el esquema ofrecido, asentado en la teoria de la ciencia de Pepper, un profesor de la universidad de Berkeley resulta atractivo, la manera de orientar, dirigir y aplicar el análisis a la psicologia de la personalidad y a su modificación, nos ha parecido más bien flojo.

${ }^{2}$ Conductismo y cognitivismo deberian entenderse en plural y no en singular, aunque pese a esta diversificación, ambas poseen como nota en común el estudio de la dinámica comportamental (y de sus posibilitantes) y un situacionismo de base. Es posiblemente este situacionismo el principal responsable del rechazo de la psicología de la personalidad que, al menos en gran medida, apela a invariantes comportamentales de media y larga duración no operacionalizables en estudios de laboratorio. 
el contexto del descubrimiento y justificación siguiendo modelos "duros" de estrategias de investigación que por el de la reformulación y aplicación a un mundo clínico que se ha visto sobrepasado por la "psicologia de la salud". ${ }^{3}$

Recientemente nos hemos ocupado de una presentación general acerca de los trastornos de personalidad y de los principales modos de acercamiento a este problema, asi como una revisión de la evidencia relevante existente y detección de algunas de las principales insuficiencias (Pelechano, de Miguel y Hernández, 1995); el objetivo de este trabajo era proponer un capitulo de introducción para un manual de psicopatología sin que se ofrecieran datos y reflexiones de corte más teórico. Por ello, podría resultar conveniente, dedicar un trabajo más bien conceptual que empírico al mismo tema, a la vista de la publicación oficial del DSMIV (cuando se escribió el capitulo del manual mencionado más arriba trabajamos a base de los borradores-hasta tres llegaron a manos del autor- elaborados como material de trabajo), de la versión española de una monografía dedicada al tema desde una perspectiva cognitiva "no oficial" (Beck, Freeman et al., 1995), así como de algunas monografías dedicadas a la personalidad y sus trastornos, en mayor o menor medida (Costa y Widiger, 1994; Heatherton y Weinberger, 1994, que refiejarían la "posición oficial de la American Psychological Association) y de algunos de los muchos puntos que quedaron sin tratar en el capítulo mencionado más arriba y que poseen cierto interés. En este sentido, las páginas que siguen deberian ser conceptualizadas como un complemento del trabajo publicado recientemente aunque cada uno de ellos posee la suficiente entidad como para poder ser leido sin referencias cruzadas.
Aparte de las razones "coyunturales" parece que el tema posee unas implicaciones considerables tanto para la clínica aplicada como para la investigación y representa, velis nolis, uno de los elementos de confluencia ( $y$ discusión, por ello mismo) entre las "especialidades" de psicología clínica y psicología de la salud, que se encuentran en liza desde hace unos años. Finalmente, puesto que desde hace una larga veintena de años estamos trabajando en la elaboración de un modelo sobre funcionamiento personal dedicaremos asimismo algún espacio a la consideración de los trastornos de personalidad desde la perspectiva y/ o modelo de parámetros (Pelechano, 1973, 1989, 1993).

Asi pues, los objetivos que pretendemos cubrir en estas páginas son los siguientes: (1) revisar y comentar algunos de los principales problemas que poseen, a nuestro juicio, las definiciones y clasificaciones de los trastornos de personalidad más comunes desde la psicopatología, insistiendo más en elementos conceptuales básicos que en la taxonomia concreta y especifica propuesta en cada caso y sus notas determinantes, junto a la presentación de una de las últimas opciones cognitivas acerca de esta conceptualización; (2)comentar los aspectos más importantes que lleva consigo la aplicación del modelo de los "cinco grandes" a la taxonomia de los trastornos de personalidad, lo que servirá como pórtico introductorio para (3) presentar el modelo de parámetros en su posible aplicación a los trastornos de personalidad más "consensuados", teniendo presente que pueden haber otros trastornos por un lado $y$, por otro, que entre los enunciados, existen redundancias y/o solapaciones serias que deberian ser tenidas en cuenta. No pretendemos con este último punto más que abrir

\footnotetext{
${ }^{3}$ La especialidad se encuentra entrecomillada y no por razones caprichosas. La psicologla clinica se ha visto sobrepasada por la psicología de la salud en la medida en que la segunda se ocuparía de las enfermedades fisicas todas, mientras que la primera se restringiria a los problemas de demencia y locura, esto es, a los problemas tipicos de la psicopatologia y psiquiatria convencionales. La psiquiatria dentro de la medicina ha tenido un desarrollo mucho menor en los últimos 50 años que las distintas especialidades de traumatologia, cirugía y medicina interna. Uno de los "pecados" de la psicología clínica serla su identificación con la psico-patología (y ésta última de corte psiquiátrico); uno de los pecados de la nueva "psicologla de la salud" sería la del olvido y hasta ignorancia de las aportaciones de la psicologla clinica a la comprensión y alivio de los problemas. Pero estos temas son motivo de otro trabajo cuya publicación puede ser inmediatamente anterior o posterior al que nos ocupa pero que ya se encuentra listo para ello (Salud, enfermedad, clinica y psicologia: un laberinto con muchos caminos ciegos, a publicar en Análisis y Modificación de Conducta, y en el que se tratan especificamente estos temas).
} 
un poco más una línea de investigación que hemos apuntado en otros lugares; abrir un poco más no significa agotar sino pergeñar y apuntar lineas de irabajo posibles, asi como denunciar aquellas otras que, desde esta perspectiva, poseen menos interés.

\section{LOS TRASTORNOS DE PERSONALIDAD DESDE LA PSICOPATOLOGÍA}

Importa menos aquí apelar a la "historia" de los trastornos de personalidad como entidad psicopatológica que llamar la atención acerca de su conceptualización ${ }^{4}$ (no quiere decir esto que despreciemos la historia, antes al contrario, pensamos que la exposición histórica, por sí misma, ya sería un trabajo; y muy largo, por cierto) en dos orientaciones distintas. Una, la mayoritaria, se encuentra recogida en la CIE-10(1992) y en el DSM-IV (1995) y representa una definición de consenso; la otra, se refiere al grupo cognitivo de Beck y Freeman (1995).

(i). Los trastornos de personalidad desde las clasificaciones consensuadas. -Las dos clasificaciones más "aceptadas" son el fruto de un consenso mayoritario entre grupos de trabajo. La CIE-10 (el capítulo V), es el sistema clasificatorio empleado por la Organización Mundial de la Salud mientras que el DSM-IV procede de la Sociedad de Psiquiatría Americana. A medida que ambas organizaciones han ido formulando sistemas más avanzados existe una mayor coherencia y compatibilidad entre ellas lo cual puede ser interpretado de dos formas: de manera positiva, como que se está logrando realmente un mayor consenso "objetivo" aunque cabe asimismo una interpretación "negativa" en la medida en que se lleva a cabo un análisis de los miembros que forman parte de las comisiones "internacionales" (de ambas organizaciones): una inspección de las mismas recoge muchas solapaciones. El consenso, entonces, es posible que se haya alcanzado a partir de un procedimiento bastante simple: que se tienda a seleccionar a las mismas personas como personas influyentes en ambas clasificaciones. Volvemos a las cuestiones básicas.

1.1. Acerca del concepto y cuestiones básicas.- En las dos clasificaciones se entiende la personalidad de una determinada manera: de manera implicita en la CIE-10 y de manera explicita en el DSM-IV se hace mención a los rasgos de personalidad. Así se puede leer en el último sistema citado: "los rasgos de personalidad son patrones persistentes ${ }^{5}$ de formas de percibir, relacionarse y pensar sobre el entorno y sobre uno mismo que se ponen de manifiesto en una amplia gama de contextos sociales y personales" (p.646). Repárese que en esta definición no aparece para nada el afecto; sin embargo, este afecto va a encontrarse presente desde el principio, a la hora de conceptualizar los trastornos.

${ }^{4}$ En lo que sigue emplearemos los años de publicación de la ClE-10 y del DSM-IV en español con el fin de homogeneizar las citas con los textos que vayan apareciendo. Repárese que por lo que se refiere a la ClE-10 los dos años coinciden. En el caso del DSM-IV existen unos meses de diferencia y de ahl la diferencia en año (1994 en original y 1995 en español; la diferencia no resulta ni estadística ni clínicamente significativa).

${ }^{5}$ No deja de resultar extraño que los rasgos, en psicología de la personalidad y en psicología diferencial, en general, son conceptos cuantitativos y dimensionales por tanto, mientras que el DSM-IV es, exclusiva y especificamente categorial (esto es, no dimensional). El autor de estas líneas y cualquier lector puede imaginar sin gran esfuerzo las discusiones que tuvieron lugar en el seno de esta comisión especial antes de la presentación-oferta de la versión definitiva de este sistema clasificatorio. Sin menoscabo de poder volver más adelante sobre la cuestion, baste la siguiente cita como para confirmar lo que acaba de mencionarse y que vendria a significar algo asi como "ni si, ni no, sino todo lo contrario": "El enfoque diagnóstico utilizado en este manual representa la perspectiva categorial de que los trastornos de la personalidad representan sindromes clínicos cualitativamente distintos. Una alternativa al enfoque categorial es la perspectiva dimensional de que los trastornos de la personalidad representan variantes desadaptativas de los rasgos de personalidad que se imbrican imperceptiblemente con la anormalidad y entre ellos mismos. Ha habido muchos intentos diferentes de identificar las dimen-siones fundamentales (...) Un modelo consiste en las cinco dimensiones siguien-tes: neuroticismo, introversión (...) rechazo o disponibilidad para experimentar, hostilidad versus cordialidad y escrupulosidad. Otro enfoque describe áreas más especificas de la disfunción de la personalidad, pudiendo incluir 15 a 40 dimensiones (...).Los grupos de trastornos de personalidad del DSM-IV (por ejemplo, raro-excéntrico, dramático-emocional y ansioso-temeroso) tambien pueden considerarse dimensiones que representan el espectro de disfunciones de la personalidad en un continuum con los trastornos mentales del Eje I. Las relaciones de los diferentes modelos dimensionales con las categorlas diagnósticas de la personalidad siguen siendo activamente investigados"(pp. 649-650 de la traducción española autorizada). 
Los trastornos de personalidad se construyen a partir de estas unidades y la idea de patología de estos rasgos poseen unas caracteristicas definidas: inflexibilidad, desadaptación, provocan un deterioro funcional significativo o un malestar subjetivo (repárese que en todos los casos se trata de un enfoque categorial y que todos los criterios se suponen de la misma importancia puesto que no se hace mención alguna a su gradación; además, resulta igualmente relevante a la hora de la determinación de criterios los "internos" o intrasujeto y los sociales o determinados por sus efectos sobre los demás). Se señalan cuatro dominios comportamentales que sirven como anclaje conceptual de análisis para la detección: cognitivo, afectivo, actividad interpersonal y control de estímulos. Se exige la presencia, al menos, de trastornos en dos de estas cuatro áreas. Finalmente, se requiere que las perturbaciones detectadas no sean debidas a efectos fisiológicos de sustancias identificadas (drogas o medicación) ni a una enfermedad médica que promueva cambios permanentes (por ejemplo, ciertos traumatismos craneales).

En la CIE-10 se defiende una idea similar: apelación a la estabilidad de la afectividad, excitabilidad, control de impulsos, formas de percibir y pensar y al estilo de relacionarse con los demás, al margen de la influencia de sustancias o enfermedad médica, desadaptación en situaciones individuales y sociales (esta " $y$ " implica que deben coincidir ambos y no como en el caso del DSM-IV, que se presenta como alternativo), aparición durante la adolescencia $e$ incluso la infancia (apelando al criterio de estabilidad, esta nota parece desprenderse por si misma, como corolario).

Parece claro, pues, que se apela fundamentalmente a una psicologia de la personalidad en la que importan los elementos inva- riantes y, por ello, la estructura aunque, curiosamente se propone una estructura categorial a la vez que se supone unos antecedentes y márgenes de tolerancia de la "normalidad" no bien delimitados-, haciendo escasa alusión a los procesos (los elementos de dinámica personal, elementos motivacionales o actitudinales, tipos de dimensiones aisladas, tanto las de orientación intrasujeto como neuroticismo o impulsividad, como las de orientación social, como autoritarismo, dogmatismo 0 antiautoritarismo).

Desde esta perspectiva, la "personalidad" refleja lo estable $y$, de ahi, lo inmutable psicológicamente. No hay nada respecto al origen de esta estructura inmodificable $y$, en todo caso, parece claro que una vez lograda esa estructura (no se dice cuándo, ni cómo), no va a poderse cambiar. El papel del aprendizaje, en esta conceptualización de personalidad es nula. Esta acepción de personalidad resulta trasnochada y difícilmente pueda ser aceptada por los psicólogo contemporáneos de la personalidad y recuerda mucho el talante de cierta psicopatología del siglo $\mathrm{XIX}$ que defendia que las alteraciones mentales eran interpretables como defectos "de carácter" fundamentalmente. $E$ inmodificables.

El caso es que los modelos estructurales de la psicología de la personalidad son dimensionales y cuantitativos, mientras que el modelo que defienden los dos sistemas clasificatorios es categorial y, más aún, rechazan explícitamente las consideraciones cuantitativas (Widiger, 1993a), con todos los problemas que ello conlleva: escaso apoyo empirico, apoyo empírico en el que se confunde la validez interna con la externa ${ }^{\hat{b}}$, exageración de similitud intragrupo en el diagnóstico, la confusión entre carencias y dificultades adaptativas y presencia de alteraciones de personalidad

\footnotetext{
- La "revisión empirica" que apoya la taxonomia propuesta tiende a restringirse a la comparación en la aplicación de los criterios diagnósticos con las clasificaciones hechas por distintos clinicos que aplican estos criterios, 10 que representa un estudio de validez interna (concordancia entre jueces) y nada dice de la validez externa que, como su mismo nombre indica, representa la utilización de criterios externos a la misma taxonomía. Esto es, por ejemplo, la emisión de predicciones de acuerdo con estos criterios externos y asumir el riesgo de que estas predicciones se cumplan o no.Como Tyrer y Ferguson (1987) recuerdan siguiendo los cánones de la psicometria: "Una buena fiabilidad no indica validez y un incremento significativo en los niveles de acuerdo en diagnóstico puede obtenerse empleando criterios insatisfactorios" (p.19)
} 
múltiples ${ }^{7}$. Estos adjetivos, pese a ser pensados originalmente para el sistema del DSMIII (Widiger, 1993b) siguen manteniéndose vigentes para el DSM-IV en la medida en que los cambios en este segundo eje tienden a ser muy pequeños en el texto de "letra grande" (de hecho, se encuentran polarizados en el número y claves de identificación de los trastornos propuestos -supresión del trastorno de personalidad pasivo-agresivo que pasa a un apéndice como uno de los aspectos a estudiar con mayor intensidad- $y$ en las notas identificativas de dos de los trastornos propuestos ya en la versión anterior: el añadido de un item para la ideación paranoide en el trastorno límite y la eliminación de dos criterios en el trastorno antisocial, junto a la unión de otros dos que antes aparecian como separados en uno solo), y sin embargo, aparecen muchos cambios "de letra pequeña" como líneas de investigación. De estas líneas nos importa una especialmente puesto que representa, asimismo, una nueva plataforma conceptual: las denominadas "estrategias de afrontamiento" (p. 767).

El estudio del afrontamiento representa una linea de investigación en psicología de la personalidad, clínica y salud que está atrayendo cada vez mayor atención de los investigadores. Uno de los orígenes de esta opción se encuentra en los propuestos mecanismos de defensa psicoanalíticos pero, a diferencia de ellos, desde la formulación de Lazarus de finales de los setenta hasta nuestros días se entienden como estrategias racionales, explicitamente planificadas por los sujetos ${ }^{8}$ para enfrentarse a los problemas que les rodean. Una fuente de discusión entre los autores se refiere al nivel de análisis al que hay que situar estas estrategias, que para el grupo de
Lazarus se trata de elementos situacionales, determinados por la situación y, en todo caso el contexto y que se modifican con el paso del tiempo; para otros autores entre los que hay que contar a Carver y al que firma este trabajo, junto a los aspectos situacionales, existen modos cronificados de afrontar situaciones en los seres humanos, que representan esfuerzos racionales en su mayor medida $y$ con poder supervivencial asimismo la mayoría de ellos (Pelechano, Matud y de Miguel, 1993). Sobre estos mecanismos y estrategias existe un considerable cuerpo de conocimientos y una diversidad de instrumentación (cuestionarios y entrevistas fundamentalmente).

El empleo que hace el DSM-IV de estas "estrategias de afrontamiento" es muy otro. Se sugiere su conceptualización como mecanismos de defensa y, por ello, la cumplimentación de una escala de mecanismos de defensa que debe emplear el clínico y que se dirige a evaluar "procesos psicológicos automáticos que protegen al individuo frente a la ansiedad y las amenazas de origen interno o externo" (p.767). Se distinguen hasta siete niveles de estos mecanismos en función de su poder adaptativo, desde un "nivel adaptativo elevado" encaminado a promover un equilibrio entre opciones conflictivas (en este nivel se inventarian procesos tales, por ejemplo, como afiliación, autoobservación y sublimación; en este nivel se llega a poseer una conciencia de las estrategias empleadas) hasta un nivel de "equilibrio defensivo" caracterizado por el fracaso de la regulación de la defensa en la contención de las reacciones del individuo ante las amenazas de origen interno o externo (la distorsión psicótica y la proyección delirante son ejemplos ilustrativos de este nivel; se trata de estrategias y modos de pro-

\footnotetext{
7 Esto es, la aparición de más de un diagnóstico de trastorno de personalidad ¿debe entenderse como la existencia de distintas personalidades en el sujeto? Pese a que por el uso que se hace de ello y de las cuestiones semánticas podría deducirse que se trataria de "personalidades múltiples", realmente de lo que se trata es de la coexistencia de distintos problemas adaptativos en el sujeto, lo que significa una cosa muy distinta. Confusión que es promovida por la denominación de los trastornos.

${ }^{8}$ Realmente esta afirmación, pese a encontrarse presente en muchos autores con-temporáneos de personalidad no acaba de recoger toda la verdad. Repárese, por ejemplo, en la estrategia de negación de cáncer que emplean muchos enfermos y que suele ir aparejada con un alargamiento en la expectativa de vida. El empleo de esta negación no es racional ni propositiva y, aunque los mecanismos no se encuentran aislados, parece corresponder a una negación explicita de la enfer-medad, pese a las evidencias que se dan en contra de esa negación. Y esta negación es rápidamente convertida en una estrategia automática.
} 
ceder inconscientes y el sujeto no llega, sin más, a aceptar que los está empleando una vez se le han detectado y denunciado, por 10 que ser trata de procesos no-conscientes). Se sugiere que la calificación respecto al mecanismo de defensa predominante acompañe al diagnóstico del trastorno de personalidad en el eje II. Pensamos que, también en este caso parece haberse perdido una gran oportunidad para acercar posiciones entre la psicopatología y la personalidad de corte más científicoexperimental.

Curiosamente, no se encuentra ninguna pista acerca de la forma en que se incardina este eje de los mecanismos de defensa con los rasgos definitorios de la personalidad que subyace a los trastornos de personalidad. Caben, al menos, dos posibilidades extremas:

(i) Que las estrategias de afrontamiento representen un sistema funcional independiente de los rasgos definitorios de los trastomos, aunque a su vez, sean, asimismo, fuentes de "otro tipo de trastornos" no especificados. Si este fuera el caso, deberían sugerirse maneras de integrar ambos sistemas 0 , al menos, de pergeñar formas de interacción.

(ii) Que las estrategias de afrontamiento formen parte de la personalidad y pueda hablarse de la caracterización de ciertas estrategias de afrontamiento en función del tipo de personalidad y/o tipo de trastorno. En este caso debería hablarse de la interacción y diversificación entre trastornos de personalidad y estilos de afrontamiento.

Ninguna de estas posibilidades es contemplada en el manual DSM-IV. Además, cabe la posibilidad de que los estilos de afrontamiento puedan ser interpretados como competencias cognitivas más que como estructuras básicamente irracionales, formando parte de la inteligencia sociopersonal (Pelechano, 1994, 1995).

1.2. Unas notas sobre la tipología propuesta.-Aparte pequeñas variaciones de situación tanto la CIE-10 como el DSM-IV permiten agrupar los trastornos propuestos en tres grandes grupos: "raro-excéntrico, dramático-emocional y ansioso-temeroso"(DSM-IV,p. 650). Lo que no aparece por ninguna parte es la justificación de esta agrupación. Si los tres grupos han sido el resultado de un análisis racional de la realidad, la presentación debería ir acompañada de las claves en función de las cuales se aceptan estos y no otros. $\mathrm{Si}$, por el contrario, este agrupamiento es fruto de un análisis histórico (los grandes síndromes de neurosis y psicosis en un grado subclínico, más un tercer grupo "mixto") deberia especificarse claramente el sentido de la misma y ofrecer las modulaciones introducidas asi como la base empirica que justifica esta clasificación; si, finalmente, lo que importa es la clasificación como tal al margen de la lógica de agrupamiento, deberia asimismo explicitarse la base empírica y las razones del agrupamiento. Por ejemplo, la agrupación de la personalidad antisocial, asimilada a cierto tipo de acciones delictivas, dentro del grupo de trastornos de personalidad "dramático-emocionales". Al autor de este trabajo le parece que toda clasificación tiene algo de caprichoso, pero si esto es asi y asimismo es reconocido por los propulsores de los DSM, no deberia ser esgrimido este argumento en contra de otras opciones que sí poseen base empirica y se han ocupado de elaborar su validez de constructo. La apelación a entidades "sub-mórbidas" no parece tener demasiada apoyatura (y menos, consensuada). Por otro lado, dos de los tres grupos parecen encontrarse relacionados con mecanismos tradicionalmente atribuidos a la ansiedad (la evitación/inhibi-ción por un lado - la exposición de uno/a mismo/a exagerada ante los demás -incluso, aun un poco traída por los pelos, el trastorno antisocial podría incluirse en este sentido, lo que resulta más un objeto de discusión que una conceptualización compartida por todos los autores; y desde luego, desde un punto de vista psicológico no existe mucha evidencia a su favor ${ }^{9}$ ).

Por otra parte, existe una redundancia considerable en los diagnósticos de los distintos trastornos. Una posible razón se encuentra en las redundancias que se dan en los elementos de diagnóstico propuestos para cada uno de

\footnotetext{
${ }^{9}$ Esto no quiere decir que no existan elementos de ansiedad en algunas "personalidades con trastorno antisocial" sino más bien que la denomianda personalidad antisocial, en el caso de existir patologia, pueden ser adscritas a un cierto poso residual psicótico del tipo de la Einheitpsychose que defendia Wernicke y que ha sido reivindicada recientemente por autores como H.J. Eysenck tras una revisión de parte, al menos, de la bibliografla existente.
} 
los trastornos. Clark, Vorhies y McEwen(1994) por ejemplo, han señalado que en el trastorno esquizoide, en el evitativo y en esquizotípico se apela a la inhibición/aislamiento social. $Y$ por el criterio elegido, basta con la existencia de unos pocos signos para la emisión de uno u otro de los diagnósticos. La razón que dan muchos autores para esta solapación es la aceptación de la diversidad entre humanos que pueden ser agrupados bajo la misma rúbrica. Sin embargo, en pura metodologia científica, lo que se pide a una clasificación es que sea útil y diferencie entre las unidades de análisis a las que se refiere. Esta utilidad y diferenciación se logra o bien a base de categorias excluyentes o bien a base de apelaciones a relaciones estructurales distintas entre las categorias propuestas, Ni una ni otra condición la cumplen las dos clasificaciones a las que estamos haciendo referencia.

En suma: las clasificaciones propuestas no parecen cumplir los requisitos mínimos que deberían poseer para que se convirtieran en criterios objetivos y fiables susceptibles del análisis científico. De hecho, tienden a aparecer algunos cambios de edición en edición. Posiblemente esta clasificación sea una muestra clara de que la propuesta sin base científica real no alcanza un consenso y, para que se obtenga un consenso de resultados el camino a recorrer sería el atenimiento al método científico en el establecimiento de conclusiones y clasificaciones.

Por otro lado, se ha recordado en alguna ocasión, que los trastornos de personalidad tienden a no ser tratados en su estado "puro" puesto que tienden a no acudir a buscar ayuda especializada. Es posible que una razón importante de ello sea la importancia que tienen en estos trastornos los elementos culturales tanto a nivel $d$ enjuiciamiento como de aceptación y tolerancia. $Y$, si esto es así, el problema se puede analizar desde el punto de vista de la propia entidad que posee, en estas clasificaciones, el concepto de tipos de "trastornos de personalidad" y sus múltiples influencias social-culturales y un considerable grado de tolerancia y permisividad que existe sobre ellos. el olvido de estos problemas disminuye el valor que poseen algunas orientaciones y trabajos, como los que se van a presentar a continuación, en los que la tipologia propuesta por los sistemas clasificatorios se emplea como criterio de contrastación y de última verdad de otras maneras de entender el problema.

(ii).La propuesta de Beck y Freeman sobre los trastornos de personalidad. - Un grupo de investigadores alrededor de Beck y Freeman (1992, traduc. 1995), siguiendo las ideas originalmente propuestas por Beck respecto a la depresión, han propuesto una teoría acerca de los trastornos de personalidad que representa, más bien, una teoría acerca de la personalidad en general, que podría ser caracterizada como cognitiva (se proclama un asentamiento en el procesamiento de información aunque no se proponen procesos concretos y modos de actuación de los mismos en los problemas que tratan), asentada en una epistemología evolucionista y de la que se derivaría una determinada forma de acción terapéutica (el cambio de creencias).

El ser humano es entendido como fruto de evolución filogenética. A lo largo de este proceso el empleo de unas formas de actuación, de motivación y de pensamiento (estrategias) han mostrado su utilidad en un momento determinado y se han consolidado hasta el punto de formar parte de su equipamiento para la supervivencia (predación, competitividad, sociabilidad, vaya por caso). Sin embargo, la evolución histórica ha promovido que algunas de estas estrategias sean adecuadas en nuestro tiempo mientras que otras se conviertan en elementos perturbadores, disfuncionales, de la integración personal y social.

Por otro lado, antes de la puesta en acción de una u otra estrategia existe un análisis de la situación (procesamiento de la información) y la evaluación de la situación es la que promueve la activación de una u otra de estas estrategias. Esta evaluación depende, en parte al menos, de las creencias que tenemos y que se refieren a esa situación. Estas creencias se encuentran insertas en estructuras más o menos estables denominadas esquemas (el concepto de esquema posee una rancia tradición en psicologia) que son las que sintetizan y seleccionan los datos que ingresan. Estas estructuras básicas que son los esquemas, de las que dependen los procesos cognitivos, afectivos y motivacionales son las unidades fundamentales de la personalidad. Los rasgos 
pueden conceptualizarse como expresiones "observables" de estas estructuras subyacentes ${ }^{10}$ : "Las pautas comportamentales que adscribimos a los rasgos o disposiciones de personalidad representan estrategias interpersonales desarrolladas a partir de la interacción entre las disposiciones innatas y las influencias ambientales" (op. cit., p.55). Así pues, atributos tales como dependencia o autonomía, que tienden a ser considerados como impulsos básicos, pueden considerarse realmente como aspectos funcionales de conglomerados de esquemas básicos. En términos comportamentales, los atributos pueden ser interpretados como "estrategias básicas".

Los trastornos de personalidad se proponen como creencias disfuncionales y desadaptativas que hacen a los sujetos vulnerables cognitivamente: asi el trastorno dependiente de personalidad representa una sensibilidad a la pérdida de amor y ayuda; el trastorno narcisista como un atentado a la autoestima. Cada creencia lleva consigo, para estos autores, la utilización de una estrategia tipica. Asi, en el trastorno paranoide, se cree que las personas son adversarios potenciales y por ello se emplea sistemáticamente una estrategia de cautela; el histriónico cree que necesita impresionar a los demás $y$, por ello utiliza sistemáticamente una estrategia "dramática"; en el trastorno antisocial, se cree que las personas existen para ser dominadas $y$, por lo tanto se emplea una estrategia de ataque hacia los demás. Un trastorno se da cuando uno o varios de los esquemas son hipervalentes $y$, por lo mismo, el umbral de activación para los subesquemas componentes es muy bajo, por lo que se activa con indicadores muy escasos o de baja intensidad; esta hipervalencia promueve, además, que sean predominantes y "colorean" los demás modos de procesamiento.
Existen ciertos problemas con esta conceptualización.

(i) De entrada habria que decir que poco añade a la psicologia de las construcciones personales de G. Kelly y, de hecho, incluso muchos de los recursos tanto interpretativos como las sugerencias de intervención se orientan en la misma dirección.

(ii) Pese a la importancia que se dice que posee el afecto en esta formulación, realmente ello se restringe a la dicotomía placer-dolor y la "reducción" cognitiva del afecto no deja de ser una dificultad que podria explicar el escaso éxito terapéutico que presenta.

(iii) La "reducción esencial" de los trastornos a sistemas creenciales y la relación entre estas creencias y las estrategias de actuación dista mucho de encontrarse de mostrada. Más aún, una de las características esenciales de estos dos niveles (creencia-actuación) es su asimetría funcional. Si se intenta asentar una conceptualización y modo de intervención sobre la relación entre creencia-sentimiento-acción deberian proponerse los caminos que diversifican, distorsionan y/o agrupan estas tres unidades ( $y$ proponemos tres para simplificar, puesto que creemos que son algunas más).

(iv) Se siguen dando por buenos los sistemas clasificatorios de corte psiquiátrico tradicional esencialmente, con alguna apelación a la falta de correspondencia entre el modelo que proponen y alguno de los trastornos de personalidad propuestos por el DSM (en concreto, el trastorno límite y el esquizotípico ${ }^{11}$ ).

\footnotetext{
10 Los autores proponen que la personalidad es una organización relativamente permanente compuesta por sistemas (estructuras entrelazadas que representan esquemas) y modalidades (maneras de interacción). Estos esquemas se organizan según sus funciones y contenido. AsÍ, los esquemas cognitivos tienen que ver con la abstracción, la interpretación y el recuerdo, "los esquemas afectivos son responsables de la generación de sentimientos; los esquemas motivacionales se relacionan con los deseos, los esquemas instrumentales preparan para la acción y los esquemas de control están involucrados en la autoobservación y la inhibición o dirección de las acciones" (op. cit., p. 69). Asimismo, en los sistemas pueden diferenciarse subsistemas (por ejemplo, en el del control, un subsistema dirigido al control personal y otro al control hacia los demás). Desde nuestro punto de vista sigue manteniéndose la expresión definitoria de personalidad como algo "relativamente permanente" $y$, al final, estos "esquemas" se identifican con los "constructos" de la psicología de G. Kelly (1955) incluso para la identificación de sus cualidades, diferenciaciones y modos de operar.

"En el caso del trastorno límite, porque tiene más que ver con el "déficit del yo" que con un contenido creencial específico; en el trastorno esquizotipico porque se trata de ciertas peculiaridades del pensamiento más que por un contenido idiosincrásico del mismo.
} 


\section{LOS TRASTORNOS DE PERSONALIDAD DESDE EL MODELO DE LOS CINCO GRANDES}

El denominado modelos de los cinco grandes representa la opción más cultivada en los últimos años, dentro del estudio acerca de la estructura de la personalidad desde una perspectiva científica. Asume la técnica de factorización como la más apropiada para la delimitación de las dimensiones básicas de la personalidad y el material del cual han partido los autores es el lenguaje cotidiano. Pese a la existencia de distintos "cinco grandes" dentro de la bibliografía especializada (Jones, Zuckermann, Costa y McCrae), es la versión de éstos últimos la que posee una mayor aceptación y es la que, después de más de una discusión, parece que está capitalizando la mayor cantidad de estudios encaminados a proponer un modelo de los trastornos de personalidad desde una perspectiva científica.

El análisis racioempírico del lenguaje proporciona una serie de facetas (equivalentes a factores "racionales" de primer orden) que, convenientemente analizadas y sometidas a análisis factorial de cierto tipo da lugar a cinco "grandes" dimensiones, que con sus facetas corresponden a las siguientes: neuroticismo (formado por ansiedad, hostilidad, depresión, desconcierto ante los demás, impulsividad y vulnerabilidad), extraversion (con las siguientes facetas evaluadas: cálido, gregarismo, asertividad, actividad, búsqueda de excitación y emociones positivas), apertura a la experiencia (formado por fantasia, estética, sentimientos, acciones posibles, ideas y valores), cordialidad (conformado por confianza, franqueza, altruismo, conformismo, modestia y mentalidad blanda) y, finalmente, escrupulosidad (competencia, orden, sentido del deber, orientación al logro, autodisciplina y deliberación).

En la medida en que se trata de un acercamiento en el que se parte del análisis del lenguaje, su hipótesis básica es que existe una proporcionalidad entre la relevancia psicológica y personal de las expresiones lingüísticas referidas a los afectos y maneras de comportarse por un lado y la frecuencia de uso de estas expresiones en el lenguaje. de esta forma la estructura obtenida recogeria el patrón de covariación propio de las expresiones más frecuentes $y$, entendiendo la frecuencia como criterio de normalidad, aquéllas que sean relevantes pero no frecuentes, deberian encontrarse ausentes de la estructura final que se obtenga (en el análisis factorial la aparición de un factor dentro de una estructura general depende de la representatividad de los ítems que lo definen y, más en concreto, de su varianza, respecto a toda la varianza que se encuentra recogida en las distintas variables, de forma que si una dimensión que sea muy relevante explica, por decirlo así, menos de un $5 \%$ de la varianza total puede perfectamente ser desestimada, a pesar de su potencial importancia clínica, como puede ser el caso de la predicción de una conducta suicida). Desde este punto de vista, el modelo de los cinco grandes podría representar, a lo sumo, las variaciones psicopatológicas lo suficientemente frecuentes dentro del universo lingüístico como para que existan respuestas abundantes y variadas a ellas. Los puntos extremos de estas variaciones, sin embargo, debido a su escasa frecuencia, no formarian parte de esta "estructura básico-lingüística".

Lo que acaba de ser dicho no se refiere a todos los modelos estructurales sino a los de los "cinco grandes" y a todos aquellos que utilizan el lenguaje como la fuente de datos original. En el modelo de H.J. Eysenck el lenguaje ocupa un lugar secundario y el primero se refiere a las tipologías de corte médico fundamentalmente que se han inventariado a lo largo de la historia de la cultura occidental, por lo que, de entrada, parecería ser más adecuado para su aplicación a la psicopatología. Desgraciadamente, la técnica de factorización utilizada por Eysenck y la lógica interna que sigue, siendo más compleja que la de los cinco grandes, impide que se puedan aceptar más de tres grandes factores temperamentales, a los que habría que añadir un factor de inteligencia general, sin apelaciones a factores de orden inferior.

Puestas así las cosas, en los últimos cinco años fundamentalmente, una serie de autores norteamericanos se han dedicado a recoger información, desde distintos puntos geográficos y con metodologias un tanto distintas acerca de la "adecuación" que poseen los cinco grandes para ofrecer una correcta descripción 
científica de los trastornos de personalidad que se encontraban recogidos en el DSM-III-R y que ahora aparecen en el DSM-IV. Una selección de autores y trabajos representativos de esta corriente se encuentra en la monografía editada por la American Psychological Asociation (Costa y Widiger, 1994). Esencialmente los resultados más relevantes pueden ser resumidos del modo siguiente:

(i) Por lo que se refiere a la estructura de cinco grandes, se ha obtenido empleando escalas de calificación cumplimentadas por familiares y conocidos y con cuestionarios autoaplicados. Estas escalas y cuestionarios, además, poseen una estimable validez convergente cuando se cumplimetan los dos para un mismo grupo de personas.

(ii) En un análisis de distintos trastornos de personalidad tienden a encontrarse relaciones significativas entre las "facetas" (o factores de primer orden) evaluados mediante cuestionarios y diagnósticos de trastornos de personalidad hechos pro expertos, si bien los coeficientes de correlación no son especialmente elevados y las muestras son, más bien, muy poco numerosas. Los análisis, sin embargo, dejan sin responder acerca de qué aspectos de los trastornos (que, como se dijo más arriba cubren actitudes, conductas, motivaciones y vida de relación) son los que presentan más relación y qué otros no, dentro de los criterios de utilización diagnóstica. O dicho de otra manera, es posible que las correlaciones encontradas sean debidas a presencia de items similares en los criterios diagnósti$\cos y$ en los cuestionarios de los cinco grandes, pero que no exista un patrón de covariación entre todos los criterios y las facetas.

(iii) De todos los factores, parece que las facetas que definen el factor de "apertura a la experiencia" poseen una importancia muy escasa en todos los trastornos identificados en el DSM-IV; por el contrario, el factor de neuroticismo parece ser el que posee mayor presencia $y$, de hecho, tienden a caracterizar muy bien el denominado "trastorno limite".

(iv) Desde un punto de vista de revisión general, posiblemente el trabajo de Widiger, Trull, Clarkin, Sanderson y Costa (1994) recogido en la monografia, sea el más ambicioso y el que recoge los tipos de resultados que, por un lado, cabria esperar a partir de los criterios diagnósticos en el DSM, por otro, las puntuaciones que cabe esperar en las facetas de los cinco grandes a partir de los rasgos asociados y enunciados por el sistema DSM y, finalmente, una revisión de los resultados empíricos obtenidos hasta el momento por la bibliografia especializada. Resulta especialmente sugerente el cuadro de la página 42 de la obra citada y cuyo comentario en el texto indica que, aunque con un status de proyecto de trabajo, existe una considerable concordancia entre ambas series de "resultados". Al autor de estas líneas, sin embargo, le sugiere una conclusión bien distinta. Asi, se preveen 132 calificaciones (entre estimadas y encontradas). De ellas no existe ninguna en la que concuerdenlos resultados provenientes de la bibliografia clinica con las previsiones que hacen los autores $y$, cuando se encuentra una coincidencia en la casilla la coincidencia va en contra de las previsiones (esto es, si se prevé una puntuación alta, se encuentra una baja, 10 que sucede en tres de las casillas). Este resultado nos parece que, precisamente, no sirve para apoyar las pre visiones del modelo de los cinco grandes.

(v) Desde un punto de vista algo distinto, cabría decir que el modelo de los cinco grandes presta un servicio considerable para la evaluación de dimensiones temperamentales de acuerdo con la frecuencia de uso en el lenguaje. Sin embargo, hay que decir que, tal y como se han definido los trastornos de personalidad en los sucesivos DSM, se incluyen elementos tempe ramentales y no temperamentales. $Y$ estos últimos tienden a no estar representados de manera adecuada en las facetas que recoge el modelo en cuestión.

(vi) Alguna reflexión más resulta de interés: una serie de resultados de la psicologia de la personalidad demuestran que el sentido y significación que poseen los resultados temperamentales se encuentra mediado, en muchas ocasiones por las competencias de corte intelectual (entre las 
que hay que contar la inteligencia evaluada mediante tests tipo $\mathrm{Cl}$ y de inteligencia general).

\section{PERSONALIDAD Y TRASTORNOS DE PERSONALIDAD DESDE EL MODELO DE PARÁMETROS}

Cuando hace ya más de dos décadas comenzamos con la presentación de este modelo, era más bien difícil encontrar autores de referencia que defendieran un esquema similar (una excepción digna de ser mencionada es la propuesta de Mcclelland (1951) acerca de la existencia de tres niveles en el estudio de la personalidad: rasgos estilísticos, esquemas cognitivos y motivos o necesidades implícitas). Poco después, desde una perspectiva de intento de síntesis teórica, el grupo de J. Royce comenzó a elaborar un modelo multinivel y muy ambicioso acerca de la individualidad que trataba de ofrecer una imagen más compleja que la que en ese momento era aceptada por la comunidad científica, modelo que se plasmó en una síntesis apretada y compleja en la monografia de Royce y Powell (1983). Y ya en esos años ochenta aparecen las propuestas de Hogan (1987) acerca de la dicotomía observador-actor y sus subsecuentes diferenciaciones y casi contemporáneamente la de McAdams $(1985,1990,1993,1994)$ sobre los tres ámbitos: rasgos disposicionales, cuestiones personales - para referirse a la temática personal, familiar y laboral- y la narrativa vital o el protagonismo del sujeto que cuenta su historia (el mundo del "yo"o sí mismo). Como un elemento complementario más, habría que decir que incluso autores como Costa y McCrae(1994) recogen una dicotomía a la hora de conceptualizar la personalidad: las denominadas tendencias básicas (en donde se encontrarian los cinco grandes) y las adaptaciones específicas, que, pese a atribuirles una importancia secundaria, cumplen un papel relevante a la hora de entender la adaptación del sujeto a su nicho ecológico. En este círculo de preocupaciones sobre lo que representa la personalidad se inscribe el modelo de parámetros y ha ido modificándose y reajustándose en función de los resultados que se han ido alcanzando; este modelo ha sido presentado en otros lugares
(Pelechano, 1973, 1989, 1991, 1993) por 10 que nos limitaremos a hacer aquí un recordatorio de los puntos que resultan de mayor interés para el objeto que nos ocupa en este trabajo.

De entrada habria que recordar algo que hemos mencionado más arriba a propósito de una limitación aparejada con el modelo de los cinco grandes: que los criterios temperamentales, tomados de modo aislado, son radicalmente insuficientes para poder llevar a cabo una evaluación de trastornos de personalidad. $Y$ menos si éstos se evalúan solamente a base de cuestionarios. $Y$ ello por dos razones: la significación funcional que poseen las dimensiones temperamentales por un lado y la naturaleza bifronte de la personalidad, por otra.

Las dimensiones temperamentales representan elementos que se encuentran situados a distinto nivel de consolidación (ver más abajo). Pero, la significación funcional que poseen depende, en gran medida de los niveles de competencia cognitiva que existan en el sujeto y en el ambiente sociocultural que le rodea. Factores tales como escrupulosidad o extraversión a nivel incluso básico, poseen unos correlatos comportamentales (unos signos externos y una sintomatologia) un tanto distintas en función de si la persona en cuestión posee niveles culturales altos o no; posee una "salida" creativa hacia la producción artística o posee unas competencias especiales en inteligencia quinestésica (esto es, posee unas capacidades muy altas para superar con éxito determinadas pruebas atléticas, jugar muy bien a uno $u$ otro deporte socialmente valorado, etc.). Por otro lado, la significación que poseen las dimensiones o rasgos evaluados son distintas en función del grado de generalidad en el que se lleve a cabo la medida: extraversión o neuroticismo, vaya por caso, puede ser evaluado a un nivel de componente situacional, to que puede resultar muy importante en un momento dado pero que tiende a perder poder predictivo a medida que la situación cambia (entre paréntesis, en la vida adulta tienden a encontrarse niveles de mayor invariancia debido, entre otras cosas, a una mayor estructura y constancia en el ambiente físico y social que rodea al sujeto que en el caso de la infancia, con lo que una parte considerable de 
lo que se atribuye como "básico" puede ser muy bien estable y consistente en la medida en que el ambiente sea estable y consistente; si no existe esta labilidad e interdependencia sujeto ambiental, la capacidad de adaptación de ese sujeto se encuentra considerablemente disminuida y puede dar que pensar en un trastorno psicopatológico grave por excesiva rigidez). Un grado similar de impulsividad posee significaciones distintas en dos sujetos que tengan distintos recursos cognitivos respecto a su control. Desgraciadamente, esta idea, que se ha encontrado reiteradamente en la bibliografia experimental y que se puede observar en la clínica, no se encuentra representada en las opciones contempladas hasta el momento y resulta esencial en el modelo de parámetros. Dentro de este modelo la formulación iría en la línea de conferir un status de parámetros a ciertas capacidades intelectuales y que son las que determinan el curso de acción y posibilidades de cambio de "rasgos casi invariantes" de la personalidad. Las habilidades interpersonales, como componentes de la inteligencia sociopersonal ocupan aquí un lugar destacado. ${ }^{12}$

El otro punto al que hemos hecho mención más arriba ha sido bautizado como "naturaleza bifronte" de la personalidad. En efecto, la estructura y dinámica personal depende tanto de uno/a mismo/a como de los demás o, dicho de otra forma, existe una consideración intrasubjetiva de la personalidad y otra social. A la hora de una evaluación de los trastornos de la personalidad deben tomarse en consideración tanto los tipos de evaluadores (uno el propio sujeto; otro, al menos, alguna persona que conviva o tenga trato intenso con él a ser posible) asi como dos tipos de dimensiones: (a) las que podrian ser denominadas propiamente intrasubjetivas tales como neuroticismo o motivación de logro por un lado $y$, por otro (b) las dimensiones sociales de la personalidad entre las que deben tomarse en consideración, por ejemplo, los valores, las visiones del mundo, dogmatismo y antiautoritarismo. Desgraciadamente este último tipo de dimensiones no ha sido tomado en cuenta por las clasificaciones al uso pese al considerable cuerpo de conocimientos que existe sobre muchas de ellas y la relevancia clinica que unas tienen $y$ otras es posible que posean aunque no han sido estudiadas ${ }^{13}$.

(i) Se defiende una idea de personalidad en la que se encuentran representados distintos niveles de análisis que van desde lo bioquímico hasta lo básico-funcional y desde las dimensiones muy resistentes al cambio hasta los elementos de reactividad situacional. La aplicación de esta conceptualización a la psicopatología implicaría una redenominación, cuanto menos, del rótulo de los "trastornos de la personalidad" tal y como éstos son empleados por las clasificaciones psiquiátricas.

(ii) Las fuentes de diferenciación se atribuyen tanto a los tipos de constructos-dimensiones-procesos aislados, como a las condiciones de estimulación, contextos de vida y/o nichos ecológicos. Por lo tanto, en la evaluación psicopatológica, así como en el estudio de su dinámica y modificación deberían diferenciarse o sugerirse, al menos, las partes correspondientes a unos y a otros elementos con el fin de romper la imagen del inmanentismo psicológico que parecen atribuir las etiquetas diagnósticas.

(iii) Se defiende una teoria general de sistemas funcionales. Se entiende que el ser humano se encuentra formado por una serie de sistemas funcionales (procesos y

\footnotetext{
12 El tema resulta especialmente relevante para la experimentación y la clínica. Hemos obtenido ya en una serie de estudios de intervención, con medidas antes, despues y tras un seguimiento de más de 9 meses (y en muestras de niños, adolescentes y ancianos) que las relaciones entre inteligencia general, habilidade interpersonales y el mundo de las actitudes se modifica a lo largo de un programa de intervención encaminado a cambiar las habilidades interpersonales o las actitudes. $Y$ este efecto no es debido a la "labilidad" o faita de fiabilidad de las medidas sino como fruto de un cambio en la estructura personal.

${ }^{13}$ Entre otras cosas, el trastorno esquizoide, caso de aceptarlo, debería ir aparejado con puntuaciones altas en dogmatismo, así como el histriónico; el antisocial deberia ser dogmático y en el trastorno de personalidad por evitación deberian encontrarse componentes importantes de antidogmatismo.
} 
dominios de funcionamiento psicológico) que son relativamente independientes en tre si y que se encuentran formados por variables que poseen un nivel de estabilidad y consistencia distinto. Las relaciones funcionales entre variables detectadas en los estudios experimentales (con control físico de variables o con control estadístico) deberian contextualizarse al sistema funcional y condiciones de obtención de los resultados, con el fin de ir perfilando cuáles son los parámetros que rigen, limitan y determinan o posibilitan estas relaciones funcionales. Los parámetros son, con frecuencia, variables pertenecientes a otro sistema y que inciden en los resultados. Una tarea importante a realizar es la de descubrir las variables que definen cada sistema funcional, asi como los parámetros relevantes que inciden en estas relaciones.

(iv) La mayoria de las asi llamadas "dimensiones básicas" de personalidad poseen su correlato en niveles de consolidación y de generalización de menor amplitud y/o cuantía. Asi, vaya por caso, extraversión puede ser evaluada a nivel de dimensiones básicas, a nivel de contextos específicos (familia, trabajo, ocio) y a nivel de reactividad situacional. En este sentido la cuestión acerca del status de los conceptosdimensiones estructurales deberia restringirse y contextualizarse respecto al objetivo que persigue el investigador y a la manera concreta de evaluar el concepto. Este punto que estamos comentando implica que se postula un continuo de estabilidades-consistencias y que la dicotomía, por ejemplo, rasgo-estado, no pasa de ser la expresión polar de este continuo.

(v) La estabilidad y consistencia de los constructos postulados como pobladores de la personalidad es una fuente de diferencias entre los sujetos. De esta forma, una alta o una baja fiabilidad no necesariamente implica que la prueba psicométrica 0 el procedimiento de medida sea malo, sino que puede reflejar la presencia de una variabilidad o de una reactividad diferencial ante la aplicación de uno u otro programa de modificación. Por lo que se refiere a la psicopatologia, es conocido que la estabilidad y consistencia de los aspectos que se consideran psicopatológicos tiende a ser más estable que el de aquellos otros que son de carácter adaptativo. Pese a que no es bien conocida la razón, pensamos que una clave interpretativa de la misma habría que buscarla en el desconocimiento que tenemos de los procesos, mecanismos, estructura y análisis de los cambios estructurales del funcionamiento psicológico "normal": si conociéramos más, podriamos conocer mejor la formación, desarrollo y modificación de las aspectos funcionales psicopatológicos (la mera reacción positiva ante una intervención terapéutica de cualquier signo que sea no es razón suficiente: la producción de un cambio mediante uno $u$ otro procedimiento tan solo nos dice que el ser humano "puede" comportarse de ese modo; otra cosa es que ese sea el modo más "connatural" de comportarse; si lo es, el cambio obtenido se mantendrá. Lo que, desgraciadamente, tiende a no ser el caso.

(vi) A la hora de hacer un diagnóstico diferencial de la personalidad deben tenerse en cuenta tanto (a) los posibles sistemas funcionales relevantes como (b) los niveles de consolidación-generalización de los sistemas de los que nos estemos ocupan do y (c) las relaciones entre las dimensiones o conceptos evaluados. En psicología de la personalidad y en esta concepción relativista, dinámica y experimental importa tantola puntuación alcanzada en los instrumentos de evaluación como las relaciones entre las distintas dimensiones evaluadas. La aparición de un trastorno no se da tan solo en la presencia de puntuaciones extremas en varias de las dimensiones sino, asimismo, en la relación que exista entre estas dimensiones evaluadas. Esto implica que las relaciones entre las variables que definen un sistema, asi como entre los distintos sistemas no son constantes y que la forma de relacionarse es uno de los elementos importantes a la hora de hacer un diagnóstico estructural. $Y$ esto que acaba de ser mencionado indicaria la necesidad por llevar a cabo estu- 
dios de ese cambio estructural y su poder de diferenciación y consolidación de los cambios a lo largo del tiempo. Asi, por ejemplo, sabemos que las diferencias en tre un joven delincuente con personalidad antisocial y otro que no lo es se encuentra no solamente en las dimensiones temperamentales (que arrojan escasas diferencias) sino, fundamentalmente, en la carencia del control de impulsividad acompañada de una puntuación alta en la percepción de los sentimientos de los demás junto a una escasa previsión de las consecuencias personales a medio plazo y una alta preocupación por las consecuencias a corto plazo de las acciones que se realizan.

(vii) Desde esta perspectiva, los denominados "trastornos de personalidad" de los sistemas clasificatorios convencionales se refieren, teóricamente, a estos "elementos básicos" a nivel de presentación programática. De hecho, sin embargo, parecen cubrir elementos situados en niveles funcionales distintos y con distinto nivel de con solidación (asi, vaya por caso, las actitudes no son todas igualmente difíciles de modificar y los pretendidos invariantes de problemas interpersonales pueden sermantenidos por invariantes situacionales interesados en el mantenimiento de estos problemas, más que por elementos de funcionamiento intrapersonal como parece ser atribuido por las clasificaciones). Desde la perspectiva de parámetros, la determinación de los comportamientos no es importante para el estudio funcional descriptivo sino para el establecimiento de los planes de modificación (ambos niveles de descripción y de modificación resultan esenciales para el modelo y su correcta comprensión).

(viii)El problema de la evaluación precisa de los trastornos de personalidad propuestos que estas clasificaciones resulta, desde aquí, más un problema de "criterio" (esto es, de la claridad, precisión, coherencia y especificación junto a la eliminación de redundancias) que de "predictor" (esto es, de la propuesta de puntuaciones sobre unas determinadas dimensiones). $Y$ en todo caso, se especifica que desde el modelo de parámetros se emplean sistemas de clasificación dimensionales y no categoriales. La apelación a la "cualidad" y cualitativo como un elemento diferencial entre lo normal y lo patológico se entiende desde parámetros no por la presencia o ausencia de un determinado elemento sino, más bien, por una distinta relación entre los elementos, en interacción con una diferente puntuación en una o más de las dimensiones propuestas. Dicho de otra manera: una fuente de variación -y por ello, de identificación de psicopatologia- puede en contrarse en la extremosidad de las puntuaciones alcanzadas sobre una serie de dimensiones; pero otra, asimismo importante puede ser no por presentar puntuaciones extremas, sino más bien por una especial relación que se dé entre las dimensiones relevantes. Mientras el sistema clasificatorio y la comprensión propuesta por el modelo de los cinco grandes -y esta afirmación sería generalizable a otros modelos dimensionales tales como los de Tellegen o el de $\mathrm{H}$. J. Eysenck- tiende a proponer solamente la primera opción que acabamos de enunciar, nosotros defendemos tanto esta primera como, lo que más nos importa, la segunda, que llama al conocimiento de la estructura personal. Reconociendo que no existen muchos estudios que hayan controlado tanto a nivel diagnóstico como de propuesta de tratamiento y de sus efectos este tipo de análisis y resultados. En nuestra experiencia esta segunda opción resulta especialmente relevante e importante para entender efectos producidos por los tratamientos, asi como para poder emitir pronósticos acerca de efectos a medio y largo plazo.

(ix) El análisis factorial asentado sobre categorias estrictamente léxicas posee una importancia escasa a la hora de estudiar elementos psicopatológicos. Desde el modelo de parámetros se propone el estudio de los elementos que se encuentran presentes, especificación de su nivel de consolidación y relevancia (que puede ser distinta para distintos grupos), correlatos operativos y su relevancia (lo situacional puede ser determinante en alguna ocasión) y 
modelos interactivos a la hora de definir las relaciones y formular predicciones.

(ix) Se diferencia entre elementos denotativos de las dimensiones, que se refieren a los aspectos significativos relevantes y los elementos valorativos, que se refieren a la aceptación y/o rechazo de esas dimensiones o procesos. Ambos aspectos deben ser tomados en consideración a la hora de la elaboración de constructos y de instrumentos.La psicología de la personalidad (y la psicopatología) se encuentra plagada de elementos valorativos que no siempre son tenidos en cuenta y que tienden a ser "eliminados" de la investigación. Frente a esta posición, se asume que existenelementos valorativos en todos los criterios. $Y$ lo que debe hacerse es diferenciar ambos aspectos, no negar uno u otro. Ansiedad, motivación de logro o locus de control, además, de que se puntúan en una escala u otra, poseen unas connotaciones positivas y negativas que deben ser tenidas en cuenta desde el comienzo y hasta el final de todo el proceso de evaluación y de interpretación.

(x) La mayor parte del mundo motivacional se interpreta en el modelo de parámetros como núcleos de factores situados en un nivel intermedio de generalización y de consolidación, por lo que pueden encontrarse elementos de uno u otro "gran" factor en estos últimos. Los componentes de movilidad o de incentivo de la acción se encuentran presentes en niveles distintos y no resulta de interés especificarlos, a serie de sistemas funcionales (procesos y este nivel de análisis, como un dominio especial, sino integrarlos dentro del modedelo más general.

(xi) En el modelo jerárquico propuesto se encuentra, en el vértice superior, el sí mismo como elemento integrador y filtro general de los distintos campos psicológicos y niveles. Este nivel más general puede ser útil para una primera estimación pero resulta radicalmente insuficiente a la hora de la programación de estudios con cretos de individuos y del establecimiento de programas de cambio. Por lo que se refiere a las dimensiones básicas relevantes para los trastornos de personalidad se diferencian entre temperamentales (neuroroticismo, extraversión, impulsividad-rigidez), creenciales (actitudes básicas y creencias sobre el funcionamiento personal, social e institucional) y competenciales (inteligencia verbal y académica, quinestésica -coordinación de movimientos gruesos y finos, espacial, de la imagen -en reposo y en movimiento, socioinstitucional -politica, histórica, económica-, y socio-personal -sabiduria, afrontamiento, interpersonal, intrapersonal-). Es en la interacción entre estos dominios en donde hay que encontrar los trastornos de personalidad.

(xii) De todo lo anterior se desprendería que una definición de trastorno de personalidad debería incorporar los niveles de consolidación y consistencia en el que se encuentran situados las características definitorias, asi como elementos de tipo cognitivo y temperamental de distintos niveles, así como de las interacciones entre las características o dimensiones y niveles que se aducen como criterios de definición. En ausencia de este tipo de especificaciones lo que quedaría por delante sería un considerable programa de investigación que se integren ordenando según el esquema pergeñado, los resultados parciales que se han alcanzado hasta el momento, se detecten las redundancias, de fectos de los estudios, maneras de corregir los sesgos y panorama de lo conocido, para pasar, posteriormente a un estudio pormenorizado y serio de aquellos elementos que desde la tradición, la bibliografía clínica publicada, las tipologias históricas y los resultados experimentales reiteradamente contrastados ( $y$ en los que hayan participado prioritariamente personal no universitario dado que del universitario tenemos casi un exceso de datos) en distintos estudios, metodología y escuelas de pensamiento. Ni el ser humano funciona como un todo organizado con consecuencias inmediatas para todas las partes si hay una que se resiente, ni funciona como las clasificaciones al uso en psiquiatría defienden, ni como los libros de psicologia, un capitulo cada vez y sin duplicaciones ni relaciones entre ellos. Búsqueda de relaciones, cambio de relaciones y puntua- 
ciones en un modelo dimensional formado por sistemas relativamente independientes parece ser un buen condimento para el guiso de unos nuevos modos de pensar acerca de los antiguamente denominados "trastornos de personalidad" y que fueron alcanzados por consenso, a través de un consenso laborioso que ha sido condenado al fracaso poco después de la publicación de estas taxonomias consensuadas.

(xii) Finalmente, más arriba se ha dicho que existe un continuo de consolidación y estabilidad. Las variables importantes en este modelo para definir uno u otro trastorno de personalidad no necesariamente necesitan encontrarse situadas en un mismo nivel de consolidación ( $y$ deseablemente como dirian los autores al respecto) en el nivel más básico. Depende de la relevancia, pertinencia y poder predictivo de la sintomatologia encontrada, que puede encontrarse asentada sobre un factor básico o varios de consolidación intermedia. Como criterio de consolidación máximo se comparan los resultados correspondientes con los obtenidos en pruebas convencionales de inteligencia (tipo inteligencia general, o tipo edad mental, por ejemplo), no todas las cuales deben ser de papel y lápiz.

A la vista de todo lo enunciado, parece claro que desde la perspectiva de parámetros se puede gestar una revisión profunda, no consensuada sino guiada por los requisitos y dictámenes de la ciencia, acerca de los trastornos de personalidad. El resultado de esta revisión ofrecería, pensamos, un panorama más rico aunque indiscutiblemente más complejo -y más cercano a la realidad, a la vezque el que se presenta en los trabajos al uso. Una revisión más pormenorizada de trastornos concretos y su enganche en los "oficialmente aceptados" así como la propuesta de otros nuevos representa un trabajo distinto al que aquí nos ha ocupado y que, con alguna probabilidad, posiblemente alguien se ocupe de ello en un futuro no muy lejano.

\section{REFERENCIAS BIBLIOGRÁFICAS}

American Psychiatric Association (1994, trad. 1995). DSM-IV. Manual Diagnóstico y estadístico de los trastornos mentales, Barcelona, Masson S.A.
Beck, A.t., Freeman, A. el al.(1995). Terapia cognitiva de los trastornos de personalidad,Barcelona, Paidós (orig. 1992, The Guilford Press).

Clark, L.A., Vorhies, L. \& McEwen, J.L. (1994). Personality disorder symptomatology from the five-factor model perspective. En P.T. Costa Jr. \& T. Widiger (eds.).-Personality Disorders and the Five-Factor Model of Personality, Washington DC., American Psychological Association.

Cook, M.(1995). Levels of personality, $2^{\text {nd }}$. edit., London, Cassell Educational Limited.

Costa, P.T. Jr. \& McCrae, R.R.(1994). Set like plaster? Evidence for the stability of adult personality. En T.F. Heatherton \& G.J. Weinberger(eds). Can personality change?, Washington DC., American Psychological Association.

Costa, P.T. \& Widiger, T.A. (eds.)(1994). Personality disorders and the Five-Factor Model of Personality, Washington DC, American Psychological Association.

Davis, R.d. \& Millon, T.(1994). Personality Change: Metatheories and Alternatives, En T.F. Heatherton \& J. K. Weinberger (eds.). Can personality change?, Washington D.C., American Psychological Association.

Heatherton, T.F. \& Weinberger, J.L. (eds.)(1994). Can Perso-nality Change?, Washington DC., American Psychological Association.

Hogan, R.(1987). Personality psychology: Back to the basics. En J. Aronoff, A.I. Rabin \& R.a. Zucker (eds.).-The emergence of personality, New York, Springer.

Kelly, G.(1955). The Psychology of Personal Constructs, vols. I y II, New York, Norton.

McAdams, D.P.(1985). Power, intimacy and the life story: Personological inquiries into identity, New York, Guilford Press.

McAdams, D.P.(1990). Unity and propose in human lives: The emergence of identity as a life story. En A.l. Rabin, R.a. Zucker, R.a. Emmons \& S. Franks (eds.). Studiyng persons and lives, New York, Springer.

McAdams,D.P.(1993). The stories we live by: Personal myths and the making of the self, New York, William Morrow.

McAdams,D.P.(1994). Can personality change? Levels of stability and growth in personality across life span. En T.F. Heatherton \& G.J.L. Weinberger (eds.). Can personality change?, Washington DC, American Psychological Association.

McClelland, D.C.(1951). Personality, New york, Holt, Rinehart \& Winston.

Millon, T.(1971). Psicopatología moderna, Barcelona, Salvat Editores S.A.

Millon, T.(1981). Disorders of personality. DSMIII:Axis II, New York, John Wiley \& Sons. 
Organización Mundial de la Salud (1992). C/E-10: Trastornos mentales y del comportamiento, Madrid, Meditor.

Pelechano,V.(1973). Personalidad y parámetros: Tres escuelas y un modelo, Barcelona, Vicens Vives.

Pelechano,V.(1989). Efes de referencia y una propuesta temática. En E. Ibáñez y V. Pelechano (eds.).-Personalidad, Madrid, Alhambra.

Pelechano,V.(1993). Personalidad. Un enfoque histórico-concep-tual, Valencia, Promolibro.

Pelechano, V., Matud, M.P. y de Miguel, A.(1993). Habilidades de afrontamiento en enfermos ffsicos crónicos, Análisis y Modificación de Conducta, 19, pp.91-149.

Pelechano, V., de Miguel, A. \& Hernández, M.(1995). Trastornos de personalidad, En A. Belloch, B. Sandín \& F. Ramos (eds). Manual de Psicopatologia, vol. II. Madrid, McGraw-Hill/Interamericana de España S.A.
Royce, J.R. \& Powell, A. Theory of personality and individual differences: Factors, systems and processes, Englewood

Tyrer, P.\& Ferguson, B. (1987). Editorial: Problems in classification of personality disorder, Psychological Medicine, 17, pp. 15-20.

Widiger,T.A.(1993a). The DSM-III-R Categorial Personality Disorder Diagnoses: A Critique and an Alternative, Psychological Inquiry, 4, pp. 7590.

Widiger,T. A.(1993b). Reply to commentators: From $B$ to $Z$, Psychological Inquiry, 4, pp. 135-141.

Widiger,T.A., Trull, T.J., Clarkin,J.F., Sanderson, C. y Costa, P.T. Jr. (1994). A description of the DSMIII-R and DSM-IV personality disorders with the five-factor model of personality. En P.T. Costa Jr. \& T.A. Widiger (eds.).-Personality Disorders and the Five-Factor Model of Personality, Washington DC, American Psychological Association. 